\title{
Acute kidney injury after coronary artery bypass graft surgery: a narrative review of causes, diagnosis, and prevention
}

\author{
MANSOUR JANNATI', A, B, D, E, JAVAD KOJURI'2, B, D, E \\ ORCID ID: 0000-0002-0908-2247 \\ ${ }^{1}$ Cardiac Surgery Ward, Namazi Hospital, Shiraz University of Medical Sciences, Shiraz, Iran \\ ${ }^{2}$ Cardiology Department, Shiraz University of Medical Sciences, Shiraz, Iran
}

A - Study Design, B - Data Collection, C - Statistical Analysis, D - Data Interpretation, E - Manuscript Preparation, F - Literature Search, G - Funds Collection

Summary Acute kidney injury (AKI) after coronary artery bypass grafting (CABG) is a common cardiac event associated with short and long-term consequences and occurs in 30-51\% of CABG patients. AKI may be associated with many other factors and is also responsible for many other pathologies. An increased level of serum creatinine $(\mathrm{SCr})$ after surgery is one of the signs of AKI that may occur more often during cardiopulmonary bypass (CPB) in susceptible individuals. Preparing preoperative checklists is a good practice for the prevention of AKI. Defining new opportunities and strategies of perioperative care is a useful procedure for decreasing the risk of AKI after cardiac surgery. Collecting more data on preoperative risk factors and improving the intraoperative practices may decrease the incidence of AKI in the aggregate population. In this review study, we are going to review the literature on the pathophysiology of AKI and introduce the discussion about the features of patients who are more at the risk of AKI than others.

Key words: coronary artery bypass, acute kidney injury, creatinine, serum, cardiopulmonary bypass.

Jannati M, Kojuri J. Acute kidney injury after coronary artery bypass graft surgery: a narrative review of causes, diagnosis, and prevention. Fam Med Prim Care Rev 2021; 23(3): 372-379, doi: https://doi.org/10.5114/fmpcr.2021.108205.

\section{Background}

Postoperative acute kidney injury (AKI) is a common postoperative complication in $30-51 \%$ of patients undergoing cardiac surgery and can increase the risk of morbidity, mortality, and the length of hospital stay [1]. Severe AKI increases the mortality rate three to eightfold and also increases the length of hospital stay and the related costs of treatment [2]. AKI is a major mortality cause of patients following cardiac surgery [3-5].

Postoperative complications have multidimensional psychological and pathological origins, including age, prior kidney injuries, "diabetes mellitus, ischemia-reperfusion injury, altered regional blood flow with vasomotor dysfunction, and inflammatory responses" [6-9]. In the case of progressive acute renal dysfunction, the survival rate is significantly lower [10]. It is also associated with a fourfold resource utilization in hospitalized individuals [11], and dramatically reduces life quality for both the patients and their families [12]

It is very important to detect patients with high-risk of progressing postoperative AKI early in order to provide them with suitable support that may include proper hemodynamic monitoring, fluid management, appropriate pharmacologic therapy, or early use of renal replacement therapy (RRT) $[13,14]$.

This paper is aimed to study the impact of postoperative coronary artery bypass graft surgery (CABG) on acute kidney injury (AKI) incidence. For this reason, some of the most reputable and well-known scientific medical resources were collected.

\section{Methods}

The relevant articles were searched in the PUBMED and SCOPUS databases. The following keywords were used: "Acute Kidney Injury," "Coronary Artery Bypass," and "Cardiopulmonary Bypass". Articles from 1990 to 2019 were searched. Ninety-five article were found, and their abstracts were read. Finally, 86 article were selected for the study.

\section{The Acute Kidney Injury (AKI) concept and definition}

There are three different classifications of AKI. After decades of no standard definition of AKI, Bellomo et al. finally introduced the RIFLE classification (Risk, Injury, Failure, Loss End-Stage Kidney Disease) in 2004 that can be seen in Table 1 [15].

In 2007, the AKIN (Acute Kidney Injury Network) group revised the RIFLE criteria. This revision introduced four main changes, namely: 1) GFR has been removed from the definition; 2 ) the $\mathrm{SCr}$ period was changed from seven days to 48 hours; 3) $0.3 \mathrm{mg} / \mathrm{dL}$ of SCr was considered as the lowest measure for $\mathrm{AKI}$, and 4) two stages (loss and end-stage) have been omitted from RIFLE. All of these changes can be found in Table 2 [16]. Finally, the KDIGO (The Kidney Disease Improving Global Outcome) workshop defined AKI according to three factors: an increase in $\mathrm{SCr}$ by $50 \%$ in seven days, an increase in $\mathrm{SCr}$ by over 0.3 $\mathrm{mg} / \mathrm{dL}$ in 48 hours, or oliguria [17].

\begin{tabular}{|c|c|c|c|c|c|}
\hline & Class risk & Injury & Failure & Loss & ESKD \\
\hline RIFLE & $\begin{array}{l}\uparrow S C r \times 1.5 \text { or } \\
\downarrow \text { GFR over } 25 \%\end{array}$ & $\begin{array}{l}\uparrow S C r \times 2 \text { or } \\
\downarrow \text { GFR over } 50 \%\end{array}$ & $\begin{array}{l}\uparrow S C r \times 3 \text { or } \\
\downarrow \text { GFR over } 75 \%\end{array}$ & $\begin{array}{l}\text { Persistent acute renal failure with } \\
\text { complete loss of kidney function } \\
\text { over four weeks }\end{array}$ & $\begin{array}{l}\text { RRT required for over three } \\
\text { months }\end{array}$ \\
\hline
\end{tabular}

EDKD - End-Stage Kidney Disease, GFR - glomerular filtration rate, RRT - renal replacement therapy, SCr - serum creatinine concentration. 


\begin{tabular}{|l|l|l|l|}
\hline Table 2. The AKIN (Acute Kidney Injury Network) classification & Stage 2 & Stage 3 \\
\hline & Stage 1 & $\mathrm{SCr}>2$ baseline & $\begin{array}{l}\mathrm{SCr}>3 \text { baseline or } \uparrow \mathrm{SCr} \text { to } 4.0 \mathrm{mg} / \mathrm{dL} \\
\text { (with an acute increase of at least 0.5 } \\
\mathrm{mg} / \mathrm{dl} \text { ) or } \uparrow \text { of RRT }\end{array}$ \\
\hline
\end{tabular}

AKIN - Acute Kidney Injury Network, RRT - renal replacement therapy, SCr - serum creatinine concentration.

\begin{tabular}{|l|l|l|l|}
\hline Table 3. The KDIGO (The Kidney Disease Improving Global Outcome) definition of AKI \\
\hline & Stage 1 & Stage 2 & Stage 3 \\
\hline KDIGO & $\begin{array}{l}\text { SCr }>1.5 \text { baseline or }>0.3 \mathrm{mg} / \mathrm{dL} \\
\text { increase }\end{array}$ & $\mathrm{SCr}>2$ baseline & $\begin{array}{l}\mathrm{SCr}>3 \mathrm{baseline} \mathrm{or} \uparrow \mathrm{SCr} \text { to } 4.0 \mathrm{mg} / \mathrm{dL} \\
\text { or } \uparrow \text { of RRT }\end{array}$ \\
\hline
\end{tabular}

RRT - renal replacement therapy, $\mathrm{SCr}$ - serum creatinine concentration.

\section{Risk markers, prevention, pathophysiology, and survival implications}

when hypotension or a low cardiac output state persists, the kidney compensatory reserves become gradually exhausted, filtration reserve is exceeded, and endogenous and/or exogenous vasopressors increase afferent arteriolar resistance that consequently reduces the GFR. At this point of pre-renal azotemia, oliguria may occur, but tubular function may remain intact. A longer period of ischemia will cause structural tubular injury with cell disruption that may obstruct the tubules with back leakage of fluid into the circulation. There are also oxidant injury and inflammatory responses that result in further hypoperfusion and damage to tubular cells $[18,19]$.

Cardiac surgery is recognized as one of the significant risk factors for postoperative AKI. It may also induce "bleeding, postoperative pro-coagulable state," and the consequent "macro thrombotic" events, including "preoperative stroke or myocardial infarction" [12]. Cardiopulmonary bypass (CPB) could accelerate microemboli formation which consists of fibrin, activated leukocytes, and aggregated platelets. Subsequently the resulted microvascular plugging with leukocytes and activated platelets may have critical roles in the pathogenesis of postoperative AKI and post-infection kidney injury [20]. However, post-CABG and $C P B$ incidence of AKI is partly associated with a platelet count drop, but still little is known about the implications due to relatively fewer comprehensive studies in a "cardiac surgical patient cohort". In addition to the increased medical costs, chronic kidney disease and dialysis, elevated hospital mortality, and a decline in long term-survival are caused by postoperative AKI [21-23]. AKI is an indicator of "all-cause mortality" in post-CABG. Consequently, it is critical in post-CABG hospitalization to develop a reliable $A K I$ prediction model for improving clinical and medical effectiveness. The number of diagnostic models for post-CABG AKI risk factors has been increasing in recent years, with "European System for cardiac operative risk evaluation (EuroSCORE)" (1999, revised in 2012) [24], and the "society of thoracic surgeons (STS) score" (2008) being the most frequently used models [25]. Another model is "The patient's age, creatinine, and ejection fraction (ACEF) score," first developed in 2009 for quick clinical tests [26]. Regardless of the frequent use of these models for postoperative mortality prediction, several preoperative "demographic and clinical variables" have also been reported as influencing postoperative mortality and morbidity caused by AKI. Evidence shows that STS scoring and database was the only most reliable test to predict endpoint renal failure [27]. The potential very limited outcomes for risk prediction model(s) may be attributed to low dialysis rates. This accounts just for $1-5 \%$ of studied patients [28]. Since AKI is a prevalent event in post-operative CABG and may cause severe outcomes, for instance, it may increase the risk of end-stage renal disease (ESRD) threefold [29], different models are required to overcome this problem. In addition, more research is needed to develop validated, reliable models to determine the severity of postoperative AKI [30].

\section{Inflammation and genetics}

Modification of genetic factors and many clinical factors is a hard or impossible task. However, other clinical risk factors for AKI could be prevented, and could help in identifying a patient with a high risk of AKI and ARF. The balance between "pro-inflammatory response and the compensatory anti-inflammatory response" is the key to modifying clinical AKI risk factors. Administration of intra-operative corticosteroids is the treatment for the systemic inflammatory response syndrome (SIRS) as an AKI risk factor [31]. However, biological active mediators of natural body compensatory anti-inflammatory response to SIRS as pro-inflammatory proteins resulting in kidney dysfunction plays a critical role in postoperative AKI prevention [32]. Inflammation is potentially caused by kidney injury in post-coronary artery bypass graft (CABG) patients [33,34], which explains "endothelial responses and neutrophil recruitment in ischemia-reperfusion injury including distant organ injury" [35] and "post-ischemic renal failure" [36]. Thrombocytopenia is a prevalent event in acute AKI patients and is attributed to elevated platelet destruction (immune and non-immune), hemodilution, platelet sequestration, and decreased production. Thrombocytopenia may be related to prolonged medical health care and hospitalization, a drop in survival rates, and an elevated incidence of AKI [37, 38].

\section{Classification}

Regarding etiology, AKI has been classified into pre-renal (reduced renal perfusion), renal (intrinsic renal insult), and post-renal (obstructive uropathy). Pre-renal etiology, followed by renal etiology are the most common causes of AKI. As volume changes are common during cardiac surgery, CSA-AKI can be divided into volume responsive and non-volume responsive. Various factors, including ischemia and ischemia-reperfusion injury, inflammation and oxidative stress, exogenous and endogenous toxins, metabolic abnormalities, and neurohormonal activation may cause renal CSA-AKI. They can be divided into three factors: hemodynamic, inflammatory, and nephrotoxic [39].

\section{Diagnosis, renal biomarkers}

There are three well-established measures for AKI diagnosing and staging; RIFLE, AKIN, and KDIGO. However, as they are based on changes in $\mathrm{SCr}$ occurring 48 hours to seven days after the original insult, they all have significant limitations for early preoperative AKI diagnosis. Another limitation is that there are various factors such as age, sex, ethnicity, muscle mass, diet, drug use, and intravascular volume loading affecting $\mathrm{SCr}$, which are not connected with kidney function and are not incorporated into these measures [40]. The other concern is that elevated creatinine levels are found only after $50 \%$ kidney function loss [41, 42]. Consequently, RIFLE, AKIN, and KDIGO are only 
instituted for postoperative diagnosis, which may be far too late for preoperative or intraoperative AKI treatment. On the other hand, urine output criteria are not a reliable measure for AKI pre-diagnosis as they do not differentiate pre-renal from intra-renal oliguria or ischemic from nephrotoxic kidney injury [43]. AKI may occur with no oliguria, and extra-renal obstruction may cause oliguria with no AKI symptoms. The $\mathrm{SCr}$ frequently drops post-CBP due to hemodilution, which can be followed by a potential delayed rise, even after GFR significantly decreases. These limitations encouraged clinicians and academics to search for new biomarkers for early AKI diagnostics to ensure timely intervention and avoid kidney function loss or failure. The number of clinical centers where renal function is evaluated using different measures than $\mathrm{SCr}$ is currently small. In the majority of cases, there is little significance of subtle changes in tubular function as long as the kidneys produce a satisfactory urine output with or without diuretics with minimal change in the SCr. For early AKI diagnostics, biomarkers should be the most sensitive means for early reliable detection. CABG could potentially raise all kidney-specific proteins such as tubular damage markers, some of which, such as neutrophil gelatinase-associated lipocalin (NGAL), cystatin C, kidney injury molecule 1 (KIM-1), and interleukin-18 (IL-18) can be indicators of early AKI, its severity and duration [44-47]. In addition, other AKI biomarkers, such as NGAL, cystatin C, KIM-1, and IL-18 are found after two to six hours post-CABG [48]. Biomarkers are more reliable early AKI indicators than SCr. Cystatin concentration indicates a more accurate baseline GFR compared to $\mathrm{SCr}$; nevertheless, NGAL is markedly increased in renal tubular cells in response to ischemic injury and detected easily in the blood and urine because of its small molecular size. Early NGAL detection is a common indicator of subsequent GFR decline; however, it is initially independent of GFR [49]. Injured distal tubule epithelial cells secrete NGAL (lipocalin-2) and enter the urine through the tubular back leak. NGAL measurement can be a consistent predictor of AKI incidence and severity. However renal baseline function significantly influences the diagnostic performance of NGAL $[50,51]$. Another tubular factor which is useful in the diagnostic differentiation between ischemic AKI and pre-renal azotemia and chronic kidney disease is KIM-1. Another AKI marker is a pro-inflammatory cytokine called IL-18, which is detectable four to six hours after CABG in the urine and peaks twelve hours after the surgery. Important outcomes such as duration of stay in the ICU and hospital, dialysis, and mortality, can be predicted by NGAL and IL-18 $[52,53]$. Cystatin C, as a low-weight-molecule protease inhibitor, facilitates predicting AKI two days before creatinine and is a reliable indicator reflecting GFR changes. There are also two new urinary cell-cycle biomarkers released by cellular stress in the tubular cell injury initiation phase (promoted by different factors such as inflammation, ischemia, oxidative stress, drugs, and toxins), called metalloproteinase-2 tissue inhibitor and insulin-like growth factor-binding protein 7 . Both are involved in the process of G1 cell-cycle arrest, which prevents cell proliferation in the case of DNA damage until the damage is repaired. They act as alarm proteins for adjacent cells in a paracrine fashion, and they can predict AKI better than NGAL, KIM-1, IL-18, or cystatin C [54]. However, their usefulness will depend on the development of a cost-effective and easy-to-use kit applicable in the surgery room or ICU. Although every single biomarker provides some predictive information on AKI incidence, a combination of biomarkers as a diagnostic panel in the early preoperative stage is required for an accurate and reliable diagnosis of AKI risk and severity [55, 56]. An ideal measure for predicting AKI within 24 hours is a noninvasive, specific, target-oriented, and sensitive biomarker which can be detected and measured in a fast and reproducible way to determine AKI risk and subtypes $[42,56]$.

\section{Prevention from acute kidney injury after CABG}

Fluid overload, electrolyte abnormalities, and metabolic acidosis are among the potential risks of chronic kidney disease.
Patients with acute renal failure (ARF) should undergo dialysis immediately before and after kidney replacement surgery. Positive fluid balance, as a kidney dysfunction risk factor, should be reduced by intraoperative hemofiltration. The mortality risk is estimated at $10 \%-15 \%$ for post-CABG patients with AKI or ARF. If applicable, the level of urea, nitrogen, and creatinine in the blood should return to baseline as part of pre-CABG clinical care. Using non-steroidal anti-inflammatory drugs, ACE inhibitors, and angiotensin II receptor blockers (ARBs) should be withheld one day prior to surgery to minimize their intra-CABG drug hypotensive effects $[57,58]$. Off-pump CABG may reduce AKI incidence; however, it has no effect on the RRT requirement [59]. Intraoperative measures should be used to try to augment renal reserve by improving renal blood flow. It elevates the GFR and prevents tubular damage in patients with renal dysfunction or with risk factors in medical history. Using heparincoated circuits or miniaturized circuits prevents intra-CABG hyperglycemia, which may decrease the systematic inflammatory response. Glomerular and tubular injury may be alleviated by leucocyte filters $[60,61]$, which consequently decrease microalbumin and creatinine-indexed retinol-binding protein. Free water and solutes may be removed by hemofiltration, which is beneficial in removing excess fluid in heart failure (HF) patients, and in improving pulmonary function. Antifibrinolytics may also minimize bleeding diathesis, which is a common event in renal dysfunction. $\varepsilon$-Aminocaproic acid is a commonly used safe agent although it is associated with some degree of renal tubular dysfunction without a significant change in creatinine clearance. Tranexamic acid is another better alternative. Aprotinin was removed from the market in 2007 because it increases renal failure risk and has other adverse side effects.

High perfusion pressure is a key AKI preventing factor, but optimal average intra-CABG arterial pressure is not yet well determined.

\section{AKI drug prevention}

The intraoperative renoprotective advantages of Nesiritide ( 6 -type natriuretic peptide), such as dilating renal afferent arterioles, and to a lesser extent, the efferent arterioles, have been demonstrated in several studies [62-65]. Showing strong natriuretic and diuretic properties, the drug, acting as a renin-angiotensin-aldosterone axis inhibitor, results in increased glomerular filtration. An initial clinical trial with human atrial natriuretic peptide in CABG patients with left ventricular dysfunction demonstrated the same protective benefits. The intraoperative administration is a bolus dose of $2 \mu \mathrm{g} / \mathrm{kg}$ over $1 \mathrm{~min}$, with a following infusion of $0.01-0.03 \mu \mathrm{g} / \mathrm{kg} / \mathrm{min}$. Fenoldopam, a selective agonist of the dopamine 1 (DA1) receptor produces a dose-dependent increase in renal plasma flow, decreases renal vascular resistance, and maintains GFR. Consequently, blood flow to the renal cortex and medulla increases, and sodium tubular reabsorption is inhibited, resulting in diuresis, natriuresis, and kaliuresis $[66,67]$. It is a CBP-CABG renoprotection drug used as a nesiritide alternative. Patients with a $\mathrm{SCr}$ over 1.4 $\mathrm{mg} / \mathrm{dL}$ should be administrated fenoldopam, a pre-CABG infusion of $0.03-0.1 \mu \mathrm{g} / \mathrm{kg} / \mathrm{min}$, and continue in the ICU for an estimated 12-hour period post-CABG. There is inconsistent data about therapeutic interventions such as preoperative statins, acetylsalicylic acid, $\mathrm{N}$-acetylcysteine, and sodium bicarbonate [68-70].

\section{Literature review}

Stallwood et al. (2004) studied acute renal failure in coronary artery bypass surgery regarding the independent effect of cardiopulmonary bypass (CPB). The statistical sample was 2199 post-CABG patients from $2000-2002$, in which patients with remarkable kidney function loss were removed using retrospec- 
tive analysis. AKI independent risk factors were analyzed using a multivariate regression analysis model. The results showed that 53 patients (2.4\%) developed AKI during hospitalization. The research also revealed $2.9 \%$ incidence of AKI in the patients who underwent on-pump surgery (among a total of 1483), compared with $1.4 \%$ in the off-pump CABG group (among a total of 716). The findings also suggested a correlation between the use of cardiopulmonary bypass and the risk of the development of acute renal failure [71].

Benedetto et al. studied miniaturized cardiopulmonary bypass and acute kidney injury among patients undergoing coronary artery bypass graft surgery. A statistical sample of this study included 705 mini-CPB (104) and conventional-CPB (601) postoperative patients. A propensity score matched-pair analysis was used to determine AKI in the patients based on the current acute kidney injury network classification. The findings revealed that 274 (38.8\%) of 705 patients developed postoperative AKI, and a total of 27 patients (3.8\%) Srequired immediate kidney replacement therapy. The study also indicated that AKI could prolong average postoperative recovery from two days for patients without AKI up to 5.4 days for patients with AKI. On the other hand, in the comparison between mini-CPB and CPB, the study revealed that the overall incidence of $A K I$ was 30 out of the 104 patients who underwent mini CPB (28.8\%), and 244 out of the 601 patients who underwent CPB $(40.5 \%)$, which indicates a remarkable decline in AKI incidence rate for mini-CPB postoperative patients [60].

Olson et al. (2012) studied acute kidney injury following coronary artery bypass surgery and long-term risk of heart failure. The study sample was a total of 24018 Swedish patients undergoing isolated CABG from 2000-2008 with no prior history of heart disease. The level of serum creatinine $(\mathrm{SCr})$ was used in this study as an indicator for AKI diagnosis in three different stages. The mean follow-up duration was 4.1 years, in which 1325 subjects (5.5\%) developed heart failure. The hazard ratios were $1.60 \%$ for stage $1 \mathrm{AKI}, 1.87 \%$ for stage $2 \mathrm{AKI}$, and $1.98 \%$ for stage $3 \mathrm{AKI}$. The results indicated a correlation between post-CABG, AKI, long-term heart failure, and kidney function loss [72].

Chen et al. (2015) compared contemporary preoperative risk models for predicting acute kidney injury after isolated coronary artery bypass grafting in a retrospective cohort study. The statistical sample of this study included 353 post-CABG patients from 2010 to 2012. This study mainly aimed to determine AKI in post-CABG patients, defined based on the Kidney Disease Improving Global Outcome (KDIGO) Clinical Practice Guideline for AKI, from 2012. The results showed that 102 patients developed AKI (28.9\%), and EuroSCORE II, STS score, and ACEF score were all good tools for diagnosing stage-3 AKI. Among them, the ACEF score performed significantly better. The study benefited from multivariate logistic regression analysis to examine the effect of CABG on AKI development. A drop in ejection fraction and elevated serum creatinine were identified as independent AKI risk factors [73].

Kertai et al. (2016) studied the relationship between platelet counts, acute kidney injury, and mortality after coronary artery bypass grafting surgery. The statistical sample of this study comprised 4217 post-CABG adult patients. To determine postoperative $\mathrm{AKI}$ and mortality, the postoperative nadir platelet counting method was used. The results showed that nadir platelet counts are a predictor of AKI and of mortality in post-CABG patients. The study also revealed a $54 \%$ overall AKI incidence rate in post-operative CABG patients. A reverse correlation was found between platelet counts and postoperative AKI. The findings also suggested an elevated mortality risk in AKI patients immediately after CABG surgery [74].

In a randomized clinical trial, Garg et al. (2017) studied kidney function after off-pump or on-pump coronary artery bypass graft surgery. They suggested that "mild or moderate increases in the serum creatinine concentration" are a significant indicator for AKI that may persist for several days. They also believe that $A K I$ is a side effect of chronic kidney dysfunction. The study aimed to classify AKI risk using randomized clinical trials and to observe differences between two AKI groups that underwent surgery either with a beating-heart technique or with cardiopulmonary bypass. The study sample contained 4752 off-pump or on-pump post-CABG patients from 79 clinical sites in 19 different countries between 2010 and 2013. The results showed a $50 \%$ risk of a AKI event (with an increase in creatinine concentration) 30 days after the surgery and a $20 \%$ risk of kidney failure after one year. The research also revealed a drop in AKI risk in $1472(17.5 \%)$ postoperative off-pump patients and in 1460 (28\%) on-pump CABG patients. The between-group difference for one-year postoperative kidney failure was not significant (17.1\% vs $15.3 \%$ ). The findings indicated that off-pump CABG surgery reduced AKI postoperative risk and could lead to a better one-year postoperative kidney function [75].

\section{Discussion}

When the kidney partly or completely stops its routine functioning with no prior symptoms, this event is called acute kidney injury (AKI). One biomarker of this condition is elevated creatinine content in the serum [76]. AKI accounts for a significant rate of post-CABG mortality, especially in the cases with the need for urgent hemodialysis $[28,77]$. Among postoperative AKI patients, mortality ranged between $7 \%$ and $38 \%[11,28,77-$ -79]. Cardiopulmonary bypass (CPB) can increase preoperative and postoperative acute renal injury. However, results regarding the collected data are inconsistent, which was partly due to different outcomes for using off-pump or on-pump CABG [80]. Dialysis is the final prescribed treatment for severe AKI leading to long-term kidney function loss [81]. However, statistical results showed up to $30 \%$ less severe AKI, which is more likely in postoperative hospitalization. The symptom may be serum creatinine concentration that is elevated more than $50 \%$ and lasts several days before it returns to the baseline levels. Among this population, $1 \%$ of postoperative patients need dialysis [82].

Coronary artery bypass grafting (CABG) is the most frequent cardiac surgery that is used as an effective treatment for angina ischemic heart disease [52]. During the last decade, the CABG incidence rate was 1000 cases per million US population. Acute kidney injury (AKI) is a common medical complication occurring in $8 \%$ to $15 \%$ post-CABG patients annually. It is associated with short and long-term mortality. A minor $0.5 \mathrm{mg} / \mathrm{dL}$ increase in serum creatinine ( $\mathrm{SCr}$ ) was shown to be correlated with 30 days elevated mortality up to 3 times more than routine $\mathrm{SCr}$ [53]. While there are several studies about moderate AKI effects on long-term kidney performance manifested by microvascular injuries, capillary density decline, and "chronic renal hypoxia," there are still gaps in this area of study [83]. On the other hand, several clinical case studies suggested a relationship between non-sever AKI and long-term kidney performance loss three months after the surgery. In the case of (on-pump or off-pump) postoperative CABG AKI, it is believed that off-pump surgery reduces postoperative AKI risk. Another important issue is whether long-term postoperative kidney function after on-pump CABG is affected after one year? According to previous studies [76, 81], there was robust evidence that off-pump CABG reduces either postoperative AKI risk immediately after surgery or kidney function three months and one year after surgery, respectively. The recent meta-analysis of 4819 randomized controlled trials revealed that off-pump CABG reduced AKI risk by up to $40 \%$ [20]. Accordingly, existing evidence indicates the reductive effect of off-pump CABG on non-sever AKI postoperative development, which appeared to be highest in preoperative CABG patients with chronic kidney disease. Nevertheless, the clear effects of one-year postoperative off-pump CABG are not yet fully known. Some studies $[84,85]$ focused on one-year postoperative effect of off-pump or on-pump CABG on AKI risk. There are some possible reasons for this shortcoming. 
While evidence for one-year postoperative off-pump reduction in AKI risk and kidney function is sufficient [85], one year may be too short a period to study all clinical and physical effects of this surgery on kidney function. The follow-up study may provide more reliable data on kidney function if it is carried out in longer durations. However, evidence showed a one-year GFR loss in off-pump CABG patients compared to on-pump surgery patients.

Since even a $20 \%$ loss in GFR measured one year after surgery does not indicate a significant change in "end-stage renal disease," serum creatinine concentration used as a major kidney function indicator may be prone to errors. The errors may be caused by in-vitro or in-situ diversity at the baseline and at the one-year postoperative follow-up which possibly results in unrecognized signal events. In addition, in some post-CABG patients, the baseline serum creatinine concentration may be unstable due to renal perfusion deficiency. For this reason, there have been systematic efforts to provide better standards for serum creatinine assay during the last decade [85]. Otherwise, AKI risk in postoperative off-pump CABG patients was evidently lower than in on-pump CABG patients, but it is still significantly higher than in other patients.

Overall findings indicated that postoperative AKI risk is at least $50 \%$ greater in post-CABG patients. The risk is correlated with elevated serum creatinine concentration during 30-day follow-up after surgery. The randomized controlled trial method was the basic diagnostic tool for postoperative AKI patients. This method started with serum creatinine concentration measurement done seven days before randomization. Then postoperative concentration was collected during hospitalization. Afterwards, the highest concentration collected during 30 days was adopted for primary analysis to confirm if AKI was diagnosed after surgery or not.

\section{Conclusions}

Post-CABG patients had up to $50 \%$ more risk of AKI compared to other patients after a 30-day follow-up. It was also evident that kidney function loss is significantly higher in the one year after CABG. However, the postoperative AKI risk and one-year kidney function loss in off-pump CABG patients was apparently lower than in on-pump GABG patients. The main findings also associated postoperative AKI after CABG with an elevated risk of long-term kidney function loss and heart failure. Accord- ing to evidence, AKI is an important predictor of long-term kidney and heart dysfunction. Moreover, a moderate $0.3-0.5 \mathrm{mg} /$ /dL increase in $\mathrm{SCr}$ indicated a significant increase in postoperative cardiac events. There is also evidence that long-term kidney function loss leads to cardiac failure. Postoperative renal failure is a key influencing factor in postoperative mortality and morbidity. Chertow et al. suggested a $63 \%$ mortality rate in postoperative hemodialysis patients due to severe AKI, compared to a $4.3 \%$ rate for patients with no AKI symptoms within 30 days after the surgery [28]. While this study was performed 15 years ago, the findings were consistent with the recent Mangano et al. study with the same mortality rates [11]. There are numerous complicated factors related to post-cardiac surgery renal failure mechanisms $[11,28,77,79]$. Using clinical variables, prediction algorithms were developed for individual postoperative AKI risk. The clinical variables were classified into four distinct areas based on their effects:

1. Factors related to renal ischemia after atherosclerosis with cardiac output reduction symptoms.

2. Exogenous nephrotoxin-induced kidney injury, such as "amino-glycoside antibiotics, diuretics, or radiologic contrast media" [80].

3. Endogenous nephrotoxin release (e.g., myoglobin, free radicals, or pro-inflammatory cytokines such as interleukin-8, interleukin-1, and tumor necrosis factor).

Baseline renal dysfunction is recognized as a primary indicator of preoperative and postoperative renal failure. Consequently, preoperative patients with an excessive creatinine level of $200 \mathrm{~mol} / \mathrm{L}$ should not be listed for CABG before their creatinine level returns to baseline concentration with renal dysfunction medical treatment. Most postoperative AKI risk factors are patient-oriented, so they are mainly beyond clinical control. The effect of CABG on AKI has been studied since the 1990s with the introduction of off-pump CABG [86]

Nesiritide (B-type natriuretic peptide), fenoldopam, nesiritide, statins, acetylsalicylic acid, $\mathrm{N}$-acetylcysteine, and sodium bicarbonate are known as common pre-, intra-, and post-CABG acute kidney injury drug prevention strategies. Intraoperative hemofiltration, adverse retention of urea, nitrogen, and creatinine in the blood to the baseline using nonsteroidal anti-inflammatory drugs, ACE inhibitors, angiotensin II receptor blockers ( $A R B s$ ) one day prior to surgery to minimize the intra-CABG drug hypotensive effects are among the other AKI prevention strategies for post-CABG patients.

Source of funding: This work was funded from the authors' own resources.

Conflicts of interest: The authors declare no conflicts of interest.

\section{References}

1. Waikar SS, Liu KD, Chertow GM. Diagnosis, epidemiology, and outcomes of acute kidney injury. Clin J Am Soc Nephrol 2008; 3(3): 844-861, doi: 10.2215/CJN.05191107.

2. Josephs SA, Thakar CV. Perioperative risk assessment, prevention, and treatment of acute kidney injury. Int Anesthesiol Clin 2009; 47(4): 89-105, doi: 10.1097/AIA.0b013e3181b47e98.

3. Mehta RH, Hafley GE, Gibson CM, et al. Influence of preoperative renal dysfunction on one-year bypass graft patency and two-year outcomes in patients undergoing coronary artery bypass surgery. J Thorac Cardiovasc Surg 2008; 136(5): 1149-1155, doi: 10.1016/j. jtcvs.2008.02.085.

4. Brown JR, Cochran RP, Mackenzie TA, et al. Long-term survival after cardiac surgery is predicted by estimated glomerular filtration rate. Ann Thorac Surg 2008; 86(1): 4-11, doi: 10.1016/j.athoracsur.2008.03.006.

5. Najafi M, Goodarzynejad H, Karimi A, et al. Is preoperative serum creatinine a reliable indicator of outcome in patients undergoing coronary artery bypass surgery? J Thorac Cardiovasc Surg 2009; 137(2): 304-308, doi: 10.1016/j.jtcvs.2008.08.001.

6. Aronson S, Fontes ML, Miao Y, et al. Risk index for perioperative renal dysfunction/failure: critical dependence on pulse pressure hypertension. Circulation 2007; 115(6): 733-742, doi: 10.1161/CIRCULATIONAHA.106.623538.

7. Loutzenhiser R, Griffin K, Williamson G, et al. Renal autoregulation: new perspectives regarding the protective and regulatory roles of the underlying mechanisms. Am J Physiol Regul Integr Comp Physiol 2006; 290(5): R1153-R1167, doi: 10.1152/ajpregu.00402.2005.

8. Fontes ML, Aronson S, Mathew JP, et al. Pulse pressure and risk of adverse outcome in coronary bypass surgery. Anesth Analg 2008; 107(4): 1122-1129, doi: 10.1213/ane.0b013e31816ba404.

9. Bellomo R, Auriemma S, Fabbri A, et al. The pathophysiology of cardiac surgery-associated acute kidney injury (CSA-AKI). Int J Artif Organs 2008; 31(2): 166-178. 
10. Metnitz PG, Krenn CG, Steltzer $\mathrm{H}$, et al. Effect of acute renal failure requiring renal replacement therapy on outcome in critically ill patients. Crit Care Med 2002; 30(9): 2051-2058, doi: 10.1097/01.CCM.0000026732.62103.DF.

11. Mangano CM, Diamondstone LS, Ramsay JG, et al. Renal dysfunction after myocardial revascularization: risk factors, adverse outcomes, and hospital resource utilization. The Multicenter Study of Perioperative Ischemia Research Group. Ann Intern Med 1998; 128(3): 194-203.

12. Ranucci M. Hemostatic and thrombotic issues in cardiac surgery. Semin Thromb Hemost 2015; 41(1): 84-90, doi: 10.1055/s-00341398383.

13. Jeske WP, Walenga JM, Menapace B, et al. Blood cell microparticles as biomarkers of hemostatic abnormalities in patients with implanted cardiac assist devices. Biomark Med 2016; 10(10): 1095-1104, doi: 10.2217/bmm-2016-0150.

14. Janati M, Mahmoodi Y, Sharifian M, et al. Prevalence and risk factors of acute renal failure after cardiac surgery in Southern Iran. Iran Red Cres Med J 2010; 2010(6): 636-639.

15. Bellomo R, Ronco C, Kellum JA, et al. Acute renal failure - definition, outcome measures, animal models, fluid therapy and information technology needs: the Second International Consensus Conference of the Acute Dialysis Quality Initiative (ADQI) Group. Crit Care 2004; 8(4): R204-R212, doi: 10.1186/cc2872.

16. Mehta RL, Kellum JA, Shah SV, et al. Acute Kidney Injury Network: report of an initiative to improve outcomes in acute kidney injury. Crit Care 2007; 11(2): R31, doi: 10.1186/cc5713.

17. Luo X, Jiang L, Du B, et al. A comparison of different diagnostic criteria of acute kidney injury in critically ill patients. Crit Care 2014; 18(4): R144, doi: 10.1186/cc13977.

18. Hudson C, Hudson J, Swaminathan M, et al. Emerging concepts in acute kidney injury following cardiac surgery. Semin Cardiothorac Vasc Anesth 2008; 12(4): 320-330, doi: 10.1177/1089253208328582.

19. Lameire N, Biesen WV, Vanholder R. Acute kidney injury. Lancet 2008; 372(9653): 1863-1865, doi: 10.1016/S0140-6736(08)61794-8.

20. Devarajan P. Update on mechanisms of ischemic acute kidney injury. J Am Soc Nephrol 2006; 17(6): 1503-1520, doi: 10.1681/ ASN.2006010017.

21. Parikh CR, Coca SG, Wang Y, et al. Long-term prognosis of acute kidney injury after acute myocardial infarction. Arch Intern Med 2008; 168(9): 987-995, doi: 10.1001/archinte.168.9.987.

22. Chen $\mathrm{TH}$, Chang $\mathrm{CH}$, Lin $\mathrm{CY}$, et al. Acute kidney injury biomarkers for patients in a coronary care unit: a prospective cohort study. PLoS ONE 2012; 7(2): e32328, doi: 10.1371/journal.pone.0032328.

23. Lin CY, Tsai FC, Tian YC, et al. Evaluation of outcome scoring systems for patients on extracorporeal membrane oxygenation. Ann Thorac Surg 2007; 84(4): 1256-1262, doi: 10.1016/j.athoracsur.2007.05.045.

24. Nashef SA, Roques F, Michel P, et al. European system for cardiac operative risk evaluation (EuroSCORE). Eur J Cardiothorac Surg 1999; 16(1): 9-13.

25. O'Brien SM, Shahian DM, Filardo G, et al. The Society of Thoracic Surgeons 2008 cardiac surgery risk models: part 2-isolated valve surgery. Ann Thorac Surg 2009; 88(1 Suppl.): S23-S42, doi: 10.1016/j.athoracsur.2009.05.056.

26. Ranucci M, Castelvecchio S, Menicanti L, et al. Risk of assessing mortality risk in elective cardiac operations: age, creatinine, ejection fraction, and the law of parsimony. Circulation 2009; 119(24): 3053-3061, doi: 10.1161/CIRCULATIONAHA.108.842393.

27. Mehta RH, Grab JD, O'Brien SM, et al. Bedside tool for predicting the risk of postoperative dialysis in patients undergoing cardiac surgery. Circulation 2006; 114(21): 2208-2216, doi: 10.1161/CIRCULATIONAHA.106.635573.

28. Chertow GM, Lazarus JM, Christiansen CL, et al. Preoperative renal risk stratification. Circulation 1997; 95(4): 878-884, doi: 10.1161/01. CIR.95.4.878.

29. Ryden L, Sartipy U, Evans M, et al. Acute kidney injury after coronary artery bypass grafting and long-term risk of end-stage renal disease. Circulation 2014; 130(23): 2005-2011, doi: 10.1161/CIRCULATIONAHA.114.010622.

30. Oezkur $M$, Wagner $M$, Weismann $D$, et al. Chronic hyperglycemia is associated with acute kidney injury in patients undergoing $C A B G$ surgery - a cohort study. BMC Cardiovasc Disord 2015; 15: 41, doi: 10.1186/s12872-015-0028-y.

31. Theriault $\mathrm{S}$, Whitlock $\mathrm{R}$, Raman $\mathrm{K}$, et al. Gene expression profiles for the identification of prevalent atrial fibrillation. J Am Heart Assoc 2017; 6(7), doi: 10.1161/JAHA.117.006057.

32. Jouan J, Golmard L, Benhamouda N, et al. Gene polymorphisms and cytokine plasma levels as predictive factors of complications after cardiopulmonary bypass. J Thorac Cardiovasc Surg 2012; 144(2): 467-473, doi: 10.1016/j.jtcvs.2011.12.022.

33. Li Z, Yang F, Dunn S, et al. Platelets as immune mediators: their role in host defense responses and sepsis. Thromb Res 2011; 127(3): 184-188, doi: 10.1016/j.thromres.2010.10.010.

34. Kornerup KN, Salmon GP, Pitchford SC, et al. Circulating platelet-neutrophil complexes are important for subsequent neutrophil activation and migration. J Appl Physiol (1985) 2010; 109(3): 758-767, doi: 10.1152/japplphysiol.01086.2009.

35. Lapchak PH, Kannan L, loannou A, et al. Platelets orchestrate remote tissue damage after mesenteric ischemia-reperfusion. Am J Physiol Gastrointest Liver Physiol 2012; 302(8): G888-G897, doi: 10.1152/ajpgi.00499.2011.

36. Singbartl K, Forlow SB, Ley K. Platelet, but not endothelial, P-selectin is critical for neutrophil-mediated acute postischemic renal failure. FASEB J 2001; 15(13): 2337-2344, doi: 10.1096/fj.01-0199com.

37. Williamson DR, Albert $M$, Heels-Ansdell $D$, et al. Thrombocytopenia in critically ill patients receiving thromboprophylaxis: frequency, risk factors, and outcomes. Chest 2013; 144(4): 1207-1215, doi: 10.1378/chest.13-0121.

38. Shehata N, Fontes ML. Thrombocytopenia in the critically ill. Can J Anaesth 2013; 60(7): 621-624, doi: 10.1007/s12630-013-9944-4.

39. Heringlake M, Schon J, Paarmann H. The kidney in critical illness: how to monitor a pivotal organ system. Best Pract Res Clin Anaesthesiol 2013; 27(2): 271-277, doi: 10.1016/j.bpa.2013.06.003.

40. Macedo E, Bouchard J, Soroko SH, et al. Fluid accumulation, recognition and staging of acute kidney injury in critically-ill patients. Crit Care 2010; 14(3): R82, doi: 10.1186/cc9004.

41. Najafi M. Serum creatinine role in predicting outcome after cardiac surgery beyond acute kidney injury. World J Cardiol 2014; 6(9): 1006-1021, doi: 10.4330/wjc.v6.i9.1006.

42. Bagshaw SM, Gibney RT. Conventional markers of kidney function. Crit Care Med 2008; 36(4 Suppl.): S152-S158, doi: 10.1097/ CCM.0b013e318168c613.

43. Koyner JL, Garg AX, Thiessen-Philbrook H, et al. Adjudication of etiology of acute kidney injury: experience from the TRIBE-AKI multicenter study. BMC Nephrol 2014; 15: 105, doi: 10.1186/1471-2369-15-105.

44. Boldt J, Wolf M. Identification of renal injury in cardiac surgery: the role of kidney-specific proteins. J Cardiothorac Vasc Anesth 2008; 22(1): 122-132, doi: 10.1053/j.jvca.2007.10.008.

45. Bennett $\mathrm{M}$, Dent $\mathrm{CL}, \mathrm{Ma} \mathrm{Q}$, et al. Urine NGAL predicts severity of acute kidney injury after cardiac surgery: a prospective study. Clin J Am Soc Nephrol 2008; 3(3): 665-673, doi: 10.2215/CJN.04010907.

46. Cruz DN, Ronco C, Katz N. Neutrophil gelatinase-associated lipocalin: a promising biomarker for detecting cardiac surgery-associated acute kidney injury. J Thorac Cardiovasc Surg 2010; 139(5): 1101-1106, doi: 10.1016/j.jtcvs.2009.11.007. 
47. Haase $M$, Bellomo R, Devarajan $\mathrm{P}$, et al. Novel biomarkers early predict the severity of acute kidney injury after cardiac surgery in adults. Ann Thorac Surg 2009; 88(1): 124-130, doi: 10.1016/j.athoracsur.2009.04.023.

48. Ristikankare A, Poyhia R, Kuitunen A, et al. Serum cystatin C in elderly cardiac surgery patients. Ann Thorac Surg 2010; 89(3): 689-694, doi: 10.1016/j.athoracsur.2009.11.018.

49. Wyckoff T, Augoustides JG. Advances in acute kidney injury associated with cardiac surgery: the unfolding revolution in early detection. J Cardiothorac Vasc Anesth 2012; 26(2): 340-345. doi: 10.1053/j.jvca.2012.01.001.

50. Mcllroy DR, Wagener G, Lee HT. Neutrophil gelatinase-associated lipocalin and acute kidney injury after cardiac surgery: the effect of baseline renal function on diagnostic performance. Clin J Am Soc Nephrol 2010; 5(2): 211-219, doi: org/10.2215/CJN.04240609.

51. Koyner JL, Vaidya VS, Bennett MR, et al. Urinary biomarkers in the clinical prognosis and early detection of acute kidney injury. Clin J Am Soc Nephrol 2010; 5(12): 2154-2165, doi: 10.2215/CJN.00740110.

52. Magro M, Garg S, Serruys PW. Revascularization treatment of stable coronary artery disease. Expert Opin Pharmacother 2011; 12(2): 195-212, doi: 10.1517/14656566.2010.517522.

53. Epstein AJ, Polsky D, Yang F, et al. Coronary revascularization trends in the United States, 2001-2008. JAMA 2011; 305(17): 1769-1776, doi: 10.1001/jama.2011.551.

54. Gocze I, Koch M, Renner P, et al. Urinary biomarkers TIMP-2 and IGFBP7 early predict acute kidney injury after major surgery. PLoS ONE 2015; 10(3): e0120863, doi: 10.1371/journal.pone.0120863.

55. Ray P, Le Manach Y, Riou B, et al. Statistical evaluation of a biomarker. Anesthesiology 2010; 112(4): 1023-1040, doi: 10.1097/ ALN.0b013e3181d47604.

56. Han WK, Waikar SS, Johnson A, et al. Urinary biomarkers in the early diagnosis of acute kidney injury. Kidney Int 2008; 73(7): 863-869, doi: 10.1038/sj.ki.5002715.

57. Rosner MH, Portilla D, Okusa MD. Cardiac surgery as a cause of acute kidney injury: pathogenesis and potential therapies. $J$ Intensive Care Med 2008; 23(1): 3-18, doi: 10.1177/0885066607309998.

58. Arora P, Kolli H, Nainani N, et al. Preventable risk factors for acute kidney injury in patients undergoing cardiac surgery. $J$ Cardiothorac Vasc Anesth 2012; 26(4): 687-697, doi: 10.1053/j.jvca.2012.03.001.

59. Seabra VF, Alobaidi S, Balk EM, et al. Off-pump coronary artery bypass surgery and acute kidney injury: a meta-analysis of randomized controlled trials. Clin J Am Soc Nephrol 2010; 5(10): 1734-1744, doi: 10.2215/CJN.02800310.

60. Benedetto $\mathrm{U}$, Luciani R, Goracci $\mathrm{M}$, E, et al. Miniaturized cardiopulmonary bypass and acute kidney injury in coronary artery bypass graft surgery. Ann Thorac Surg 2009; 88(2): 529-535, doi: 10.1016/j.athoracsur.2009.03.072.

61. Bolcal C, Akay HT, Bingol H, et al. Leukodepletion improves renal function in patients with renal dysfunction undergoing on-pump coronary bypass surgery: a prospective randomized study. Thorac Cardiovasc Surg 2007; 55(2): 89-93, doi: 10.1055/s-2006-924571.

62. Chen HH, Sundt TM, Cook DJ, et al. Low dose nesiritide and the preservation of renal function in patients with renal dysfunction undergoing cardiopulmonary-bypass surgery: a double-blind placebo-controlled pilot study. Circulation 2007; 116(11 Suppl.): I134-I138, doi: 10.1161/CIRCULATIONAHA.106.697250.

63. Mentzer RM, Jr., Oz MC, Sladen RN, et al. Effects of perioperative nesiritide in patients with left ventricular dysfunction undergoing cardiac surgery: the NAPA Trial. J Am Coll Cardiol 2007; 49(6): 716-726, doi: 10.1016/j.jacc.2006.10.048.

64. Dyke $\mathrm{CM}, \mathrm{Bhatia} \mathrm{D}$, Aronson $\mathrm{S}$, et al. Perioperative nesiritide and possible renal protection in patients with moderate to severe kidney dysfunction. J Thorac Cardiovasc Surg 2008; 136(5): 1369-1370, doi: 10.1016/j.jtcvs.2007.12.079.

65. Beaver TM, Winterstein AG, Shuster JJ, et al. Effectiveness of nesiritide on dialysis or all-cause mortality in patients undergoing cardiothoracic surgery. Clin Cardiol 2006; 29(1): 18-24.

66. Meco M, Cirri S. The effect of various fenoldopam doses on renal perfusion in patients undergoing cardiac surgery. Ann Thorac Surg 2010; 89(2): 497-503, doi: 10.1016/j.athoracsur.2009.09.071.

67. Barr LF, Kolodner K. N-acetylcysteine and fenoldopam protect the renal function of patients with chronic renal insufficiency undergoing cardiac surgery. Crit Care Med 2008; 36(5): 1427-1435. doi: 10.1097/CCM.0b013e31816f48ba.

68. Mithani S, Kuskowski M, Slinin Y, et al. Dose-dependent effect of statins on the incidence of acute kidney injury after cardiac surgery. Ann Thorac Surg 2011; 91(2): 520-525, doi 10.1016/j.athoracsur.2010.10.061.

69. Bolesta S, Uhrin LM, Guzek JR. Preoperative statins and acute kidney injury after cardiac surgery: utilization of a consensus definition of acute kidney injury. Ann Pharmacother 2011; 45(1): 23-30, doi: 10.1345/aph.1P384.

70. Patel NN, Rogers CA, Angelini GD, et al. Pharmacological therapies for the prevention of acute kidney injury following cardiac surgery: a systematic review. Heart Fail Rev 2011; 16(6): 553-567, doi: 10.1007/s10741-011-9235-5.

71. Stallwood MI, Grayson AD, Mills K, et al. Acute renal failure in coronary artery bypass surgery: independent effect of cardiopulmonary bypass. Ann Thorac Surg 2004; 77(3): 968-972, doi: 10.1016/j.athoracsur.2003.09.063.

72. Olsson D, Sartipy U, Braunschweig F, et al. Acute kidney injury following coronary artery bypass surgery and long-term risk of heart failure. Circ Heart Fail 2013; 6(1): 83-90, doi: 10.1161/CIRCHEARTFAILURE.112.971705.

73. Chen SW, Chang CH, Fan PC, et al. Comparison of contemporary preoperative risk models at predicting acute kidney injury after isolated coronary artery bypass grafting: a retrospective cohort study. BMJ Open 2016; 6(6): e010176, doi: 10.1136/bmjopen-2015-010176.

74. Kertai MD, Zhou S, Karhausen JA, et al. Platelet counts, acute kidney injury, and mortality after coronary artery bypass grafting surgery. Anesthesiology 2016; 124(2): 339-352, doi: 10.1097/ALN.0000000000000959.

75. Garg AX, Devereaux PJ, Yusuf S, et al. Kidney function after off-pump or on-pump coronary artery bypass graft surgery: a randomized clinical trial. JAMA 2014; 311(21): 2191-2198, doi: 10.1001/jama.2014.4952.

76. Kellum JA, Lameire N, et al. Diagnosis, evaluation, and management of acute kidney injury: a KDIGO summary (Part 1). Crit Care 2013; 17(1): 204, doi: org/10.1186/cc11454.

77. Conlon PJ, Stafford-Smith M, White WD, et al. Acute renal failure following cardiac surgery. Nephrol Dial Transplant 1999; 14(5): 1158-1162.

78. Andersson LG, Ekroth R, Bratteby LE, et al. Acute renal failure after coronary surgery - a study of incidence and risk factors in 2009 consecutive patients. Thorac Cardiovasc Surg 1993; 41(4): 237-241, doi: 10.1055/s-2007-1013861.

79. Corwin HL, Sprague SM, DeLaria GA, et al. Acute renal failure associated with cardiac operations. A case-control study. J Thorac Cardiovasc Surg 1989; 98(6): 1107-1112.

80. Gamoso MG, Phillips-Bute B, Landolfo KP, et al. Off-pump versus on-pump coronary artery bypass surgery and postoperative renal dysfunction. Anesth Analg 2000; 91(5): 1080-1084, doi: 10.1213/00000539-200011000-00007.

81. Lo LJ, Go AS, Chertow GM, et al. Dialysis-requiring acute renal failure increases the risk of progressive chronic kidney disease. Kidney Int 2009; 76(8): 893-899, doi: 10.1038/ki.2009.289.

82. Rosner MH, Okusa MD. Acute kidney injury associated with cardiac surgery. Clin J Am Soc Nephrol 2006; 1(1): 19-32, doi: 10.2215/ CJN.00240605.

83. Basile DP. The endothelial cell in ischemic acute kidney injury: implications for acute and chronic function. Kidney Int 2007; 72(2): 151-156, doi: 10.1038/sj.ki.5002312. 
84. Siew ED, Peterson JF, Eden SK, et al. Outpatient nephrology referral rates after acute kidney injury. J Am Soc Nephrol 2012; 23(2): 305-312, doi: 10.1681/ASN.2011030315.

85. James MT, Ghali WA, Tonelli M, et al. Acute kidney injury following coronary angiography is associated with a long-term decline in kidney function. Kidney Int 2010; 78(8): 803-809, doi: 10.1038/ki.2010.258.

86. Patel NC, Grayson AD, Jackson M, et al. The effect off-pump coronary artery bypass surgery on in-hospital mortality and morbidity. Eur J Cardiothorac Surg 2002; 22(2): 255-260, doi: 10.1016/S1010-7940(02)00301-9.

Tables: 3

Figures: 0

References: 86

Received: 25.08 .2020

Reviewed: 06.09.2020

Accepted: 22.09 .2020

Address for correspondence:

Mansour Jannati, MD

Cardiac Surgery Ward

Namazi Hospital

Shiraz University of Medical Sciences

Shiraz

Iran

Tel.: +98-9171026985

E-mail: mansour.jannati@mail.com 